Recherches amérindiennes au Québec

\title{
L'invisibilisation autochtone dans les politiques coloniales et républicaines
}

Le cas des Mantas-Huancavilcas (région littorale de l'Équateur)

The Invisibility of the Coastal Indigenous Groups in Colonial and Republican Policies

The Case of Mantas-Huancavilcas of the Southern Ecuadorian

Coast

\section{La invisibilización indígena en las políticas coloniales y republicanas}

\section{El caso de los Manta-Huancavilcas (región costera de Ecuador)}

\section{Vildan Bahar Tuncay}

Volume 48, numéro 1-2, 2018

URI : https://id.erudit.org/iderudit/1053718ar

DOI : https://doi.org/10.7202/1053718ar

Aller au sommaire du numéro

\section{Éditeur(s)}

Recherches amérindiennes au Québec

\section{ISSN}

0318-4137 (imprimé)

1923-5151 (numérique)

Découvrir la revue

\section{Citer cet article}

Tuncay, V. B. (2018). L'invisibilisation autochtone dans les politiques coloniales et républicaines : le cas des Mantas-Huancavilcas (région littorale de l’Équateur). Recherches amérindiennes au Québec, 48(1-2), 201-211. https://doi.org/10.7202/1053718ar
Résumé de l'article

Cet article traite de l'invisibilisation des groupes autochtones du littoral de l'Équateur dans les politiques coloniales et républicaines. Cette invisibilisation se manifeste de diverses façons : par l'absence de ces groupes dans les politiques d'assimilation, d'acculturation et d'exploitation de l'époque coloniale et la faible présence des missions religieuses dans le littoral, de même que par l'absence de ces groupes dans les politiques indigénistes des années 30 et 40 et leur association à l'acculturation et à l'assimilation à la société métisse. À partir de la fin des années soixante, une série de phénomènes a mis en évidence les limites de la stratégie volontaire d'invisibilisation comme pratique de résistance et la nécessité de structurer un discours identitaire différent afin de protéger les terres communales. Il s'agit de l'émergence des conflits relatifs au statut légal des terres communales et aux enjeux de fragmentation et de vente des terres, et du développement des activités touristiques dans la région.
Tous droits réservés @ Recherches amérindiennes au Québec, 2018

Ce document est protégé par la loi sur le droit d'auteur. L'utilisation des services d'Érudit (y compris la reproduction) est assujettie à sa politique d'utilisation que vous pouvez consulter en ligne.

https://apropos.erudit.org/fr/usagers/politique-dutilisation/ 


\author{
Vildan Bahar \\ Tuncay \\ Département de \\ géographie, \\ Université Laval, \\ Québec
}

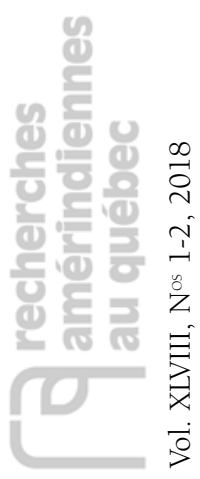

D FFÉRENTS FACTEURS sont associés à l'invisibilisation ou à l'absence de certains groupes autochtones latino-américains par rapport à d'autres dans les politiques visant leur exploitation, assimilation et intégration. Pendant la période coloniale, les régions difficiles d'accès situées en périphérie et ayant peu ou pas de potentiel de main-d'œuvre - celles qui sont peu hospitalières à l'établissement humain en raison des pluies abondantes, des mangroves envahies de moustiques, des marais et de la forêt tropicale-sont restées en marge du système d'exploitation colonial. Il s'agit, notamment, du nord du Plateau central mexicain, des terres de la haute Amazonie, de la région de Darién et de la région septentrionale du littoral équatorien faisant partie de la zone biogéographique de Chocó. Les populations natives qui s'y étaient établies ont subi à un degré moindre les effets du système, comparativement à celles qui étaient localisées dans les vallées centrales de l'Empire aztèque ou dans les hauts plateaux situés sur l'axe nord-sud de la cordillère des Andes.

Pendant la période républicaine, parmi les facteurs d'invisibilisation figurent :

- une population plus ou moins nombreuse dispersée sur des territoires étendus comme les jungles amazoniennes du Pérou (Stocks 1978 : 18) et de l'Équateur (Esvertit Cobes 2008) ou l'épaisse jungle tropicale de Darién au Panama;
- la localisation dans une région qui ne présente pas un potentiel d'exploitation des ressources naturelles;

- les pratiques d'isolement volontaire exercées par certains groupes comme les Tarahumaras du nord du Mexique et les Tagaeris d'Amazonie équatorienne.

Dans ce contexte, le présent article traite de l'invisibilisation des MantasHuancavilcas du littoral méridional de l'Équateur dans les politiques coloniales et républicaines. Cette invisibilisation se manifeste chronologiquement par:

- l'absence de ces groupes dans les politiques d'assimilation, d'acculturation et d'exploitation de l'époque coloniale;

- la faible présence des missions religieuses dans le littoral par rapport à leur implantation dans les communautés andines dès le début de la période coloniale et dans les communautés amazoniennes dès ledébut de la période républicaine;

- l'absence de ces groupes dans les politiques indigénistes des années 30 et 40 ;

- l'association de ces groupes à l'acculturation et à l'assimilation à la société métisse dans un contexte marqué par les relations conflictuelles entre l'État équatorien et les groupes andins et amazoniens à partir des années 70 .

La première section de cette étude étudie la place des Mantas-Huancavilcas 


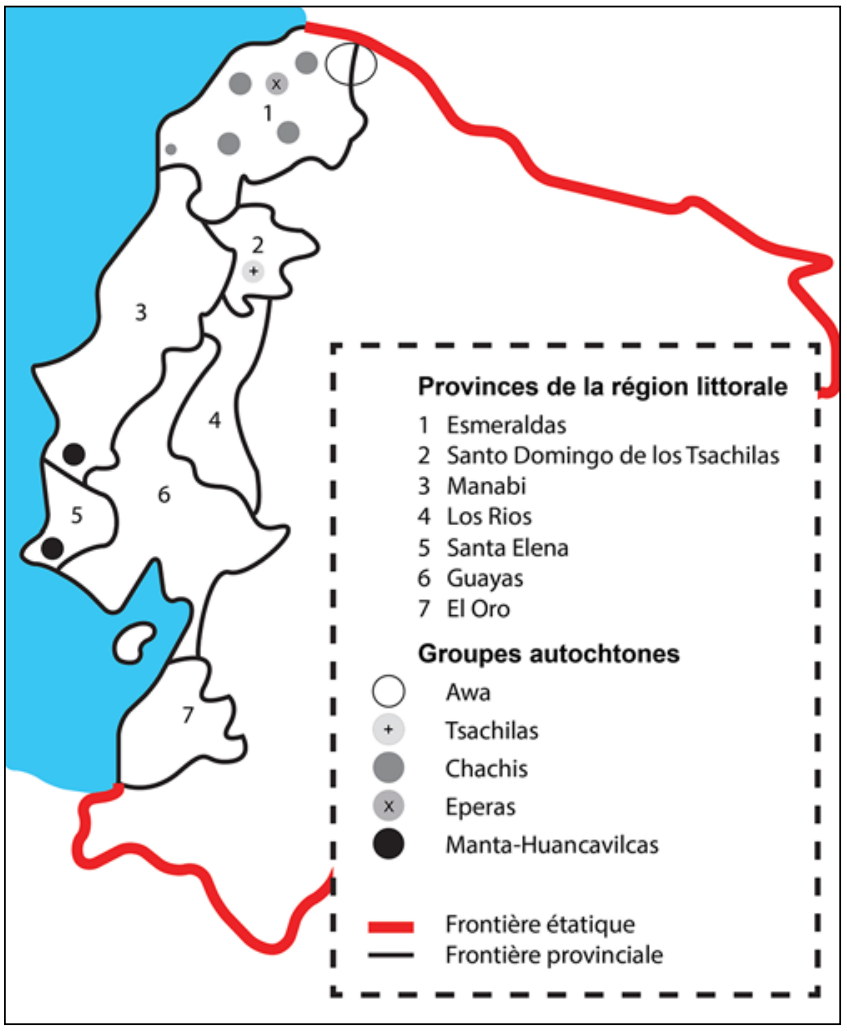

Figure 1

Localisation géographique des groupes autochtones du littoral équatorien

dans l'administration économique et sociale coloniale, en se basant sur les chroniques coloniales, les sources secondaires concernant les exercices de recensement coloniaux et l'intégration de l'Audience royale de Quito dans l'économie coloniale.

La deuxième section traite de l'invisibilisation des MantasHuancavilcas dans la distribution territoriale des missions religieuses, dans les exercices de recensement républicains et dans les politiques indigénistes des années 30 et 40 .

La troisième section aborde deux études de cas qui analysent l'invisibilisation des Mantas-Huancavilcas sous l'administration du président Rafael Correa (2007-2017). Il s'agit de l'analyse des attitudes des acteurs étatiques et du secteur privé face aux stratégies des autochtones du littoral visant à empêcher la fragmentation et la vente des parcelles communales. En revanche, la transformation de l'identité indienne vers l'identité chola à travers le temps et l'absence de marqueurs identitaires reconnus par l'État ont été utilisées comme argument par les acteurs ayant intérêt à l'achat de ces terres. La deuxième étude de cas concerne la place accordée à ces groupes dans les stratégies étatiques de marketing touristique visant la mise en tourisme du littoral et des cultures préhispaniques.

\section{Groupes autochtones dU LitTORAL MÉRIDIONAL DANS L'ADMINISTRATION ÉCONOMIQUE ET SOCIALE COLONIALE}

La région littorale équatorienne compte deux sousrégions (septentrionale et méridionale) aux caractéristiques géographiques différentes et ayant connu des évolutions historiques dissemblables concernant leur intégration à la nation équatorienne. Comme l'expose le croquis ci-dessous,

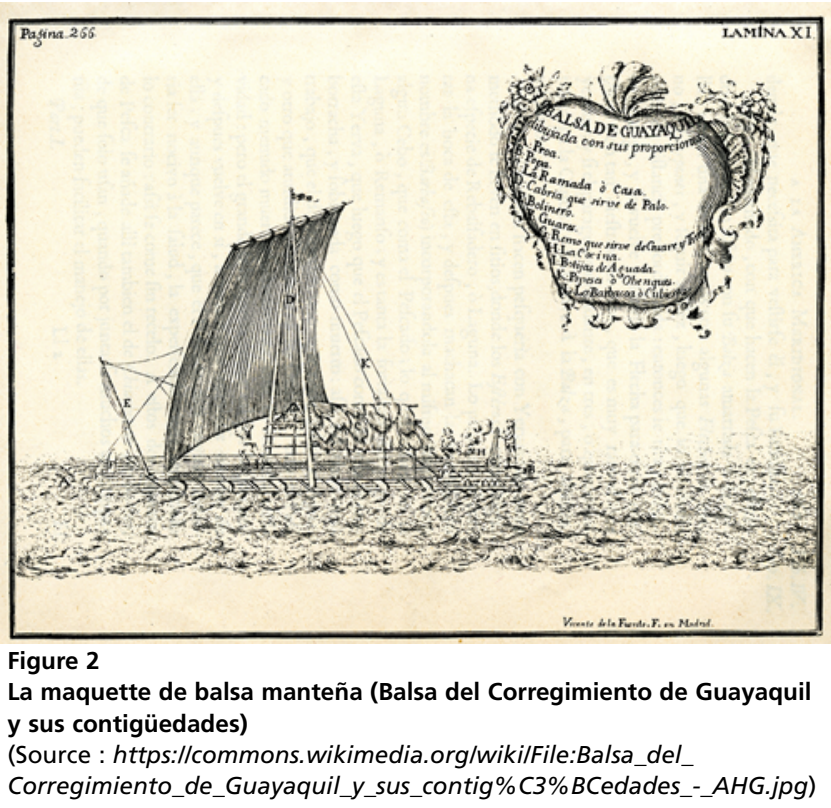

les Awas, les Chachis et les Eperas vivent dans la province d'Esmeraldas située dans la région septentrionale du littoral, et les Mantas-Huancavilcas dans la région méridionale du littoral. Ils sont répartis entre les provinces de Santa Elena et Manabi. Les Tsachilas quant à eux, sont localisés à 500 mètres au-dessus du niveau de la mer et font partie de la nouvelle province de Santo Domingo de los Tsachilas.

À l'arrivée des conquistadors espagnols au Nouveau Monde, les Manteños (600-1532), ancêtres des MantasHuancavilcas, étaient des peuples navigateurs, dont la survie dépendait des ressources marines et des produits agricoles. Dans de grands radeaux appelés balsas, ils transportaient et commercialisaient des spondylus (coquilles servant d'offrandes dans les rituels), du coton et de la laine. Les Huancavilcas (ou Manteños du Sud) vivaient dans la région qui correspond actuellement à la péninsule de Santa Elena.

Soumis aux Incas à partir du règne de Huayna Capac (? 1467 -? 1525), les Mantas-Huancavilcas sont restés à l'écart de leur domination centralisatrice étant donné que la cosmovision inca se structurait autour de la cordillère des Andes. La région littorale de l'Équateur actuelle se situait à sa périphérie dans une perspective tant géographique (Deler 2007: 193, 195) que symbolique. Le croquis cidessous est basé sur le mapamundi du chroniqueur incaespagnol Felipe Guaman Poma de Ayala (? 1550 -? 1616) élaboré au tout début de la période coloniale. Il expose la localisation des différentes villes selon une hiérarchisation symbolique par rapport à leur distance de Cuzco, considéré comme le centre du monde. Dans cette représentation symbolique qui ne respecte pas la localisation géographique objective des lieux, le littoral équatorien (port de Guayaquil) se voit attribuer une place à l'extrême ouest de Cuzco, ce qui démontre sa situation périphérique dans les imaginaires politiques incas.

Malgré les variétés démographiques et culturelles caractérisant les populations préhispaniques du Nouveau Monde, les conquistadors espagnols utilisaient le terme générique 
d'Indiens (Indios) en raison de l'erreur commise par Christophe Colomb qui croyait avoir atteint les Indes. Théoriquement, les habitants constituaient des sujets libres de la Couronne espagnole. En pratique, au vu des distances géographiques et de la soif d'enrichissement des conquistadors, régnait un système de travail forcé dans des conditions déplorables. Or, plusieurs cédules royales avaient interdit les mauvais traitements qui étaient infligés aux Indiens, incluant l'esclavage. Jumelée aux impacts des épidémies successives du Xvi ${ }^{e}$ siècle, cette situation explique les chutes démographiques importantes du début de l'époque coloniale. Les populations natives étaient intégrées au système économique colonial en tant que réserve de main-d'œuvre bon marché dans différents types d'activités telles que l'exploitation minière, l'élevage, la culture de la canne à sucre et du blé (Wolf 1962 : 158-162).

Dans le cadre de cette administration coloniale, la région d'étude faisait partie de l'Audience royale de Quito fondée en 1563 comme division administrative rattachée à la Vice-royauté du Pérou. La population de la région septentrionale de l'Empire inca qui correspond sensiblement à l'Équateur d'aujourd'hui s'était déjà amoindrie durant l'expansion militaire inca. Par la suite, la population a chuté d'environ $65 \%$ entre 1561 et 1590 en raison des conflits avec les conquistadors et des maladies comme la rougeole et la variole (Tyrer 1988 : 26-27).

Dépourvu d'une main-d'œuvre susceptible d'être exploitée, le littoral n'a pas subi les effets du système d'encomiendas ${ }^{2}$ avec la même intensité que la région andine. En effet, la ville de Guayaquil, fondée en 1537, a été conçue par les autorités coloniales comme un port nécessaire dans le littoral pacifique (Roberts 2010 : 23). En 1560 , elle ne comptait que treize encomenderos disposant d'une main-d'œuvre indienne peu nombreuse, ce qui explique la faible accumulation de richesses par les encomenderos du littoral comparativement à leurs pairs andins (Fauría 1995 : 392-393). En bref, les encomenderos étaient peu motivés à délaisser les commodités de la région andine et à s'installer dans cette zone périphérique de l'Empire colonial (Álvarez 2002 : 59; Austin Alchon 1991 : 13). Du milieu du $\mathrm{XVI}^{\mathrm{e}}$ siècle jusqu'au XvII (début de la production cacaoyère), les activités d'extraction du bois et la construction navale constituaient les principales activités économiques du littoral (Pareja et Paredes 2006 : 35). Les Indiens y jouaient un rôle primordial, étant donné que les bateaux espagnols étaient mal adaptés à la navigation près des côtes et des embouchures des fleuves. Les populations natives de cette région assuraient vers Guayaquil le transport fluvial du bois extrait à l'intérieur du littoral (León Borja 1988 : 205).

Pendant cette période, il était important pour les autorités coloniales de déterminer le nombre d'Indiens puisque ces derniers payaient le tribut et s'investissaient dans les travaux forcés. Ainsi, les recensements se réalisaient en termes pratiques d'exploitation plutôt qu'en termes socioculturels. Les calculs ont été effectués dans un premier temps par les conquistadors et les chroniqueurs; plus tard, par des vice-rois ou des fonctionnaires espagnols en visite pour recueillir des données sur les ressources de l'Empire, lesquelles étaient compilées dans les Relaciones geográficas de Indias. Les registres tenus pour l'Audience royale de Quito comprenaient des données relatives au nombre et à la 


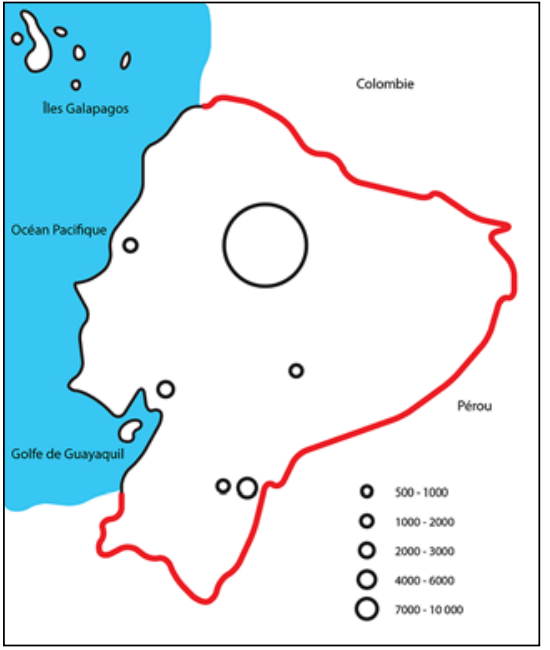

Figure 4

Répartition de la population indienne des régions littorale et andine payant le tribut en 1561

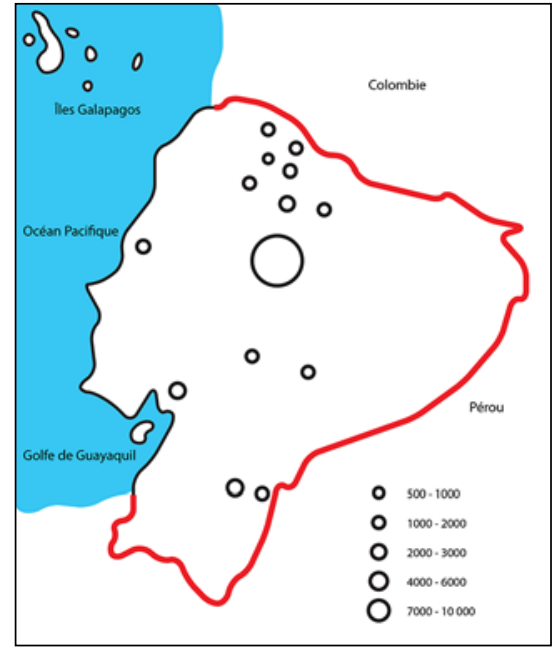

Figure 5

Répartition de la population indienne des régions littorale et andine payant le tribut en 1591

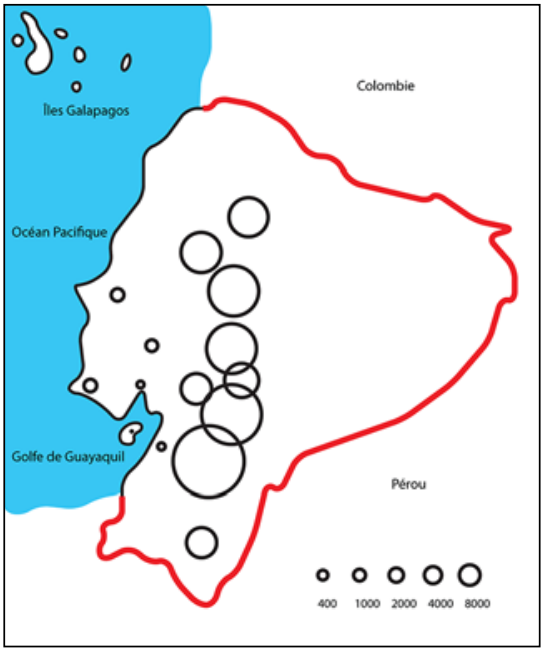

Figure 6

Population indienne des régions littorale et andine selon le recensement colonial de 1778-1780

\begin{tabular}{|c|c|c|c|}
\hline \multicolumn{4}{|c|}{$\begin{array}{c}\text { Tableau } 2 \\
\text { Population indienne des principaux établissements } \\
\text { andins 1561-1591 }\end{array}$} \\
\hline ÉTABLISSEMENTS ANDINS / ANNÉE & 1561 & 1570 & 1591 \\
\hline $\begin{array}{l}\text { Quito } \\
\text { Population totale }\end{array}$ & 240670 & - & - \\
\hline Indiens soumis au tribut & 48134 & 42000 & 24380 \\
\hline $\begin{array}{l}\text { Cuenca } \\
\text { Population totale }\end{array}$ & - & - & - \\
\hline Indiens soumis au tribut & 8000 & 8000 & 1472 \\
\hline $\begin{array}{l}\text { Loja } \\
\text { Population totale }\end{array}$ & - & - & - \\
\hline Indiens soumis au tribut & 3647 & 6000 & 2849 \\
\hline $\begin{array}{l}\text { Zamora } \\
\text { Population totale }\end{array}$ & - & - & - \\
\hline Indiens soumis au tribut & 6093 & 5000 & 685 \\
\hline \multicolumn{4}{|c|}{$\begin{array}{l}\text { (Sources : compilation de l'auteure à partir de Deler }(2007: 201) \text { basé sur les chroni } \\
\text { ques de Juan Lopez de Velasco (1574); Tyrer (1988: } 27) \text { basé sur les compilations } \\
\text { par Luis de Morales Figuera (1591) des visites du vice-roi Garcia Hurtado de } \\
\text { Mendoza effectuées en 1571) }\end{array}$} \\
\hline
\end{tabular}

localisation des Indiens sur les territoires qui correspondent aujourd'hui à l'Équateur, essentiellement en fonction du paiement du tribut.

En se basant sur ces données, il est possible de cartographier la répartition de la population indienne payant le tribut entre 1561 et 1591 selon les régions géographiques ${ }^{3}$. Force est de constater que les données des figures 4 et 5 varient en fonction des relaciones consultées, et les variations sont parfois significatives et contestables, compte tenu de la proximité des dates de production.

De ces informations, il ressort que la démographie indienne du littoral n'a pas connu de variation significative entre 1561 et 1571, mais a enregistré une baisse importante vers la fin du xvl siècle en raison des épidémies de variole et rougeole qui ont décimé presque un tiers de la population des régions andine et littorale (Laviana Cuetos 1987 : 145).
La population totale des territoires qui correspondent à l'Équateur selon le recensement colonial 1778-1780 (Empadronamiento de 1780) varie aussi en fonction des sources consultées. Selon Knapp (1987: 27) et selon les données compilées par Sánchez Parga (1996: 17), la population totale s'élevait à 412000 habitants, dont $64 \%$ étaient composés d'Indiens, $26 \%$ de Blancs et $11 \%$ gente libre de varios colores ${ }^{4}$, d'esclaves et de mulâtres. Selon Paz y Miño (1942), la population totale atteignait plutôt 342559 pour un total de 222618 Indiens, dont 213287 vivaient dans la région andine et 9331 dans la région littorale (Paz y Miño 1942 : 35). Les Indiens de la région andine constituaient $68 \%$ de la population alors que ceux de la région littorale constituaient $30 \%$ de la population. Pour terminer, d'après Hamerly (1973), la population des mêmes territoires s'élevait à 446966 habitants. Le pourcentage de la population indienne de l'ancienne province de Guayaquil représentait $30 \%$ de la population totale, soit le même que celui de l'étude de Paz y Miño (1942). La figure 6 présente la répartition des Indiens dans les principaux établissements humains des régions andine et littorale à la fin du xviII ${ }^{e}$ siècle.

La comparaison des figures 4, 5 et 6 met en évidence la récupération démographique des Indiens du littoral au xVIII ${ }^{\mathrm{e}}$ siècle, surtout celle des villes de Portoviejo, Montecristi et Jipijapa. À part ces trois régions, de plus petits groupes étaient concentrés dans les villages de Canoa, Chone, Balao, Machala et Pasaje ainsi que dans la frange côtière d'Esmeraldas. À l'île de Puno - où vivaient 10000 Indiens au début de la colonisation espagnole - ne résidaient plus que quelques dizaines de familles. Il est à noter que les Tsachilas et les Chachis n'étaient pas intégrés dans les exercices de recensement du XVIII ${ }^{e}$ et du XIX ${ }^{e}$ siècle.

\section{FAIBLE PRÉSENCE DES GROUPES AUTOCHTONES DU LITTORAL DANS LES POLITIQUES RÉPUBLICAINES}

Les indépendances du milieu du XIX ${ }^{e}$ siècle n'ont pas changé le statut socio-économique et politique des Indiens. Malgré les idées émancipatrices des libertadores pour 
leur accorder les mêmes droits que les autres citoyens, les hiérarchies sociales coloniales ont perduré. Au sein des républiques oligarchiques, les élites issues de l'aristocratie criolla ayant succédé à l'aristocratie coloniale considéraient les masses indiennes comme une main-d'œuvre disponible, soumise et presque gratuite. Sous l'effet du libéralisme économique et du capitalisme émergents, les inégalités se sont consolidées, notamment par la désintégration des terres collectives et par la reconversion des Indiens en ouvriers agricoles ou en petits propriétaires terriens (Barre 1983 : 28-29). Les Indiens se sont retrouvés exclus des bénéfices du développement économique et des services en matière de santé et d'éducation, en plus d'être sans droit de vote du fait d'être analphabètes.

Pour le cas de l'Équateur, dès le milieu du $\mathrm{xIX}^{\mathrm{e}}$ siècle, l'exportation cacaoyère constituait un des piliers de l'économie de la jeune république et elle était concentrée dans la région littorale. Étant donné sa relative faiblesse démographique et sa main-d'œuvre indienne insuffisante par rapport à la demande, un nombre important de travailleurs agricoles andins ont migré vers les provinces de Manabi et de Guayas, ce qui a transformé les dynamiques démographiques par un métissage accéléré non seulement entre les Indiens et les Blancs, mais aussi avec les Afro-équatoriens (Álvarez 1997: 100-101). Inversement, dans le sud de Manabi et dans la péninsule de Santa Elena (une région aride, sans possibilité d'irrigation et peu propice à l'agriculture), jusqu'à la fin des années 1800, on a observé la quasi-absence de métissage biologique entre ces mêmes groupes (Álvarez 1997 : 100-101; Portais 1990 : 12).

Cette même période correspond aussi à l'implantation des missions religieuses comme stratégie de l'État équatorien pour intégrer certains groupes indiens à la jeune nation. Cette stratégie ciblait essentiellement les groupes amazoniens, lesquels, depuis l'époque coloniale, disposaient d'une grande autonomie sur des territoires étendus et inaccessibles (Taylor 1991 : 112; Esvertit Cobes 2008: 20-21). Ainsi, l'État équatorien prévoyait combler la quasi-absence de l'administration publique par une administration religieuse ayant pour but de convertir les Indiens amazoniens en citoyens équatoriens. Alors que la documentation sur les missions religieuses en Amazonie équatorienne est abondante, aucune donnée n’a été repérée sur ces missions dans la région d'étude, sauf une référence aux misiones Lauritas (Álvarez 2002 : 181).
Tableau 3

province de Guayaquil 1605-1801

\begin{tabular}{|c|c|c|c|c|c|c|}
\hline ANNÉE & \multicolumn{3}{|c|}{ GUAYAQUIL } & \multicolumn{2}{c|}{ PORTOVIEJO } & \multicolumn{2}{c|}{$\begin{array}{c}\text { INDIENS SOUMIS AU TRIBUT- } \\
\text { (PROVINCE DE GUAYAQUIL) }\end{array}$} \\
\hline & $\begin{array}{c}\text { TOTAL DE LA } \\
\text { POPULATION } \\
\text { INDIENNE }\end{array}$ & $\begin{array}{c}\text { INDIENS SOUMIS } \\
\text { AU TRIBUT }\end{array}$ & $\begin{array}{c}\text { TOTAL DE LA } \\
\text { POPULATION } \\
\text { INDIENNE }\end{array}$ & $\begin{array}{c}\text { INDIENS SOUMIS } \\
\text { AU TRIBUT }\end{array}$ & $\begin{array}{c}\text { TOTAL DE LA } \\
\text { POPULATION } \\
\text { INDIENNE }\end{array}$ & $\begin{array}{c}\text { INDIENS SOUMIS } \\
\text { AU TRIBUT }\end{array}$ \\
\hline $\mathbf{1 6 0 5}$ & 2576 & 657 & 1461 & 358 & 4037 & 1015 \\
\hline $\mathbf{1 6 6 2}$ & - & 350 & - & 190 & 2160 & 540 \\
\hline $\mathbf{1 7 5 4}$ & 3000 & - & 2000 & - & 5000 & - \\
\hline $\mathbf{1 7 5 6}$ & - & 635 & - & 555 & - & 1190 \\
\hline $\mathbf{1 7 6 5}$ & 5343 & 1051 & 2540 & 636 & 7883 & 1687 \\
\hline $\mathbf{1 7 7 8}$ & - & - & - & - & 8985 & - \\
\hline $\mathbf{1 7 8 0}$ & 5972 & - & 3350 & - & 9322 & - \\
\hline $\mathbf{1 7 8 5}$ & - & - & - & - & - & 2149 \\
\hline $\mathbf{1 7 8 6}$ & - & 1844 & - & - & - & 2646 \\
\hline $\mathbf{1 7 9 0}$ & 7592 & 1796 & 4135 & 785 & 11727 & 2581 \\
\hline $\mathbf{1 7 9 6}$ & - & 1724 & - & - & - & 2556 \\
\hline $\mathbf{1 8 0 1}$ & 7600 & 1801 & 4800 & 925 & 12376 & 2726 \\
\hline
\end{tabular}

(Sources : compilation de l'auteure à partir de Deler (2007: 201) basé sur les chroniques de Juan Lopez de ce-roi Garcia Hurtado de Mendoza effectuées en 1571; Laviana Cuetos, 1987 : 143) basé sur les Actes de Cabildo de Guayaquil (1662 et 1754), les rapports de visitador Sarratea (1756) et de Zelaya (1765); Hamerly $(1973: 65,80)$ basé sur les données du recensement colonial 1778-1780 dans les Archives nationales de Quito)

\section{Tableau 4} par canton, 1840

\begin{tabular}{|l|c|c|c|c|}
\hline Canton / composition ethnique & Blancs-Métis & Indiens & Mulâtres & Noirs (en Condition d'esclave) \\
\hline Daule & 29,6 & 41,4 & 28,3 & 0,7 \\
\hline Santa Elena & 2,2 & 96,9 & 0,8 & 0,1 \\
\hline Baba & 46,4 & 8,3 & 44,5 & 0,8 \\
\hline Babahoyo & 32,0 & 19,0 & 45,1 & 3,9 \\
\hline \multicolumn{5}{|l}{ (Source : compilation de l'auteure à partir des données de Hamerly $1973: 91)$} \\
\hline
\end{tabular}

Dans un autre ordre d'idées, les réflexions sur les causes de la stagnation économique des années 30 et 40 et le besoin de potentialiser la main-d'œuvre indienne ont constitué les fondements de l'indigénisme politique, influent au Mexique et dans les pays andins. Pour la consolidation des marchés nationaux, en créant des producteurs et des consommateurs, l'intégration des masses rurales et indiennes dans le domaine économique et culturel était extrêmement importante. Au-delà des préoccupations économiques, le dénominateur commun des différentes versions de l'indigénisme était l'objectif d'encadrer et de contrôler des populations indiennes et de résorber l'altérité indienne dans la trame de la nationalité (Favre 1996: 8). Cependant, l'indigénisme n'est pas seulement un mouvement d'idées à expression politique, il est aussi un mouvement social, littéraire et artistique qui positionne l'Indien dans le cadre d'une problématique nationale (Favre 1996: 7).

Dans ce contexte, une des caractéristiques principales de l'indigénisme équatorien est la reconnaissance des conditions sociales et économiques des Indiens (Martinez 1916 : 211-212) en raison desquelles la très grande majorité 
ne pouvait devenir ni producteur ni consommateur au bénéfice de l'économie nationale (Jaramillo Alvarado 1941 : 459). Labsence d'éducation chez les Indiens, qui, selon la Dirección nacional de estadísticas, représentaient en $193957 \%$ d'une population de trois millions (Saintoul 1988 : 28), aurait non seulement conduit à ce qu'ils deviennent un obstacle au développement national, mais expliquerait également leur non-participation dans la vie économique et politique du pays. Léducation faciliterait l'intégration nationale puisque d'après les élites, les Indiens éduqués deviendraient presque automatiquement des métis (Sinardet 1999a: 133-134).

Un autre élément important dans la réflexion indigéniste équatorienne est le lien établi entre les conditions de vie des Indiens et certaines caractéristiques qui leur sont attribuées : être serviles, tristes, dociles, paresseux, manquer de sens de la compétition et consommer de l'alcool (Martinez 1916:213-214; Chavez 1943 : 581 ; Chiriboga 1939 : 625; Áquiles Perez 1943 : 6-7, 55), ces caractéristiques étant considérées comme les principaux facteurs explicatifs du retard économique du pays (Martinez 1916 : 216; Áquiles Perez 1943 : 37).

Dans ces travaux, les préoccupations pour l'hygiène se démarquent, étant donné que, selon les élites politiques, la saleté et la maladie sont les marqueurs des différences sociales alors que l'hygiène est un indicateur de modernité et de civilisation (Colloredo Mansfield 1998 : 187). La décision d'établir et d'imposer des règles d'hygiène minimales dépasse la santé et la fortification du corps pour inclure l'éducation physique et sexuelle des enfants, le développement harmonieux de la psychologie et la réalisation des études anthropométriques sur les corps indiens (Sinardet 1999b : 412).

Force est de souligner que c'est principalement l'Indien andin (et non l'Indien amazonien ou l'Indien du littoral) qui était au cour des travaux indigénistes. Dans toute la documentation consultée, la seule référence aux groupes du littoral est celle de Chávez Gonzalez (1935), qui parle pour les années 30, d'un métissage rapide et bénin de ces groupes avec les premières générations de conquistadors espagnols. Il parle également de l'adoption, par les groupes du littoral, de certaines pratiques associées aux Blancs (ex. équitation) et de l'usage de l'espagnol comme langue de communication (ibid. : 331-333).

Dans cette recherche de solution aux problèmes socioéconomiques et dans la planification des politiques sociales, les données des recensements étaient des instruments essentiels pour les autorités étatiques (Angosto Ferrandez et Kradolfer 2012 : 2-3). Cependant, comme l'État ne disposait pas des moyens nécessaires, le premier recensement national moderne dans le pays s'est organisé plus tard, en 1950. Le critère retenu pour distinguer les différentes catégories ethniques était la langue d'usage des citoyens de 6 ans et plus. Le questionnaire catégorisait les bilingues en quatre groupes, selon la langue maternelle et la langue seconde: espagnol-langue indienne; langue indienne-espagnol; espagnol-langue étrangère et langue étrangère-espagnol. L'Indien est celui qui parle uniquement une langue indienne à domicile ou celui qui est bilingue et dont la première langue apprise est une langue indienne. Les langues indiennes mentionnées dans le questionnaire étaient le quechua, le jibaro, le cayapa et le záparo (Saunders $1961: 17$ ).

Les résultats sont différents selon la source consultée. D’après Saintoul (1988), les Indiens constituaient $40 \%$ de la population totale, qui s'élevait à un peu plus que trois millions (Saintoul $1988: 29$ ); d'après l'Instituto nacional de estadisticas y censos (INEC), ce pourcentage est de $13,6 \%$ pour une population totale de 3202757 habitants (INEC 2006 : 16), et d'après Saunders, il est de 16,3\%.

Dans cette nouvelle représentation de la réalité sociale équatorienne qui propose comme critère d'indianité l'usage d'une langue indienne, les Mantas-Huancavilcas disparaissent des représentations statistiques de l'indianité. De plus, la province d'Esmeraldas voit sa population indienne augmenter progressivement sous l'effet des migrations forcées des Awas et des Eperas qui parlaient l'awapit et le siapede, respectivement.

Le questionnaire du recensement de 1962 ne faisait aucune référence à l'indianité. Celui de 1990 intègre le terme " indigène » (indígena), un terme plus neutre qu' « indien », chargé de sens et de connotations négatives selon les contextes dans plusieurs pays latino-américains (Salazar Soler 2013 : 50). De plus, à partir du début des années 80 , les représentants des différentes organisations régionales s'auto-identifiaient dans leurs discours comme indígenas.

Le questionnaire de 1990 a suscité de nombreuses contestations. La question sur la langue d'usage à domicile entre les membres de la famille obligeait les répondants à faire un seul choix entre l'espagnol, une langue indigène ou une langue étrangère. Le choix entre bilinguismes espagnol -langue indigène ou langue indigène-espagnol n'était plus possible. Il n'y avait pas de choix proposé pour la langue indigène, le répondant la précisait dans la sous-question. Selon les résultats obtenus, pour une population totale de 9648189 habitants, 362500 personnes ont déclaré une langue indigène comme langue d'usage à domicile, ce qui constituait 3,8 \% de la population équatorienne (Sánchez Parga 1996 : 18).

En 2001, l'auto-identification s'introduit comme critère d'indigénéité, en plus de l'usage d'une langue indigène. Le questionnaire proposait comme catégories : indigène, blanc, 
noir/Afro-Équatorien, métis, mulâtre et autre. Le répondant qui choisissait la première précisait la nationalité ou le peuple $e^{5}$ indigène auquel il s'identifiait. À cette date, la péninsule de Santa Elena n'avait pas encore obtenu le statut de province et faisait partie de la province de Guayas recevant un nombre important de migrants. En conséquence, les Mantas-Huancavilcas se sont fondus dans la masse indigène de cette province.

Le dernier recensement de la population a été réalisé en 2010. Les questions 14, 15 et 17 de la quatrième section concernaient la langue d'usage du répondant et de ses parents, l'autoidentification selon la culture et les coutumes ainsi que selon le peuple ou la nationalité indigène auxquels celui-ci s'identifie. Durant les préparatifs du recensement, les représentants des organisations communales ${ }^{6}$ de la péninsule de Santa Elena ont revendiqué l'intégration du terme comunero dans les catégories proposées puisqu'ils ne s'identifiaient pas à la typologie proposée. Plus précisément, ils souhaitaient que les autorités ajoutent « Vous considérez-vous comme comunero » dans le questionnaire. Cette demande n'a pas été reçue favorablement. Le plus grand pourcentage de la population qui s'autoidentifie comme indigène dans la région septentrionale du littoral vit dans la province d'Esmeraldas. Selon ces résultats, la péninsule de Santa Elena, qui concentre le plus grand nombre de communes de la région (68), compte 1,35\% de personnes s'auto-identifiant comme telles (Álvarez 2016 : 339) par rapport à 79,13\% s'auto-identifiant comme métis.

\section{OUI À L'AUtOCHTONE du PASSÉ ET NON À L'AUTOCHTONE DU PRÉSENT : L'INVISIBILISATION des Mantas-Huancavilcas à l'époque DU CORREÍSMO (2007-2017)}

À l'invisibilisation dans les catégorisations des recensements de population s'ajoutent les réactions des acteurs étatiques aux stratégies des groupes du littoral visant à empêcher la fragmentation ou la vente des parcelles communales à la propriété privée. Principalement, les autorités étatiques et les représentants du secteur privé utilisent l'absence de marqueurs identitaires autochtones reconnus par l'État et l'argument d'acculturation de ces groupes pour la non-reconnaissance de certains droits dont bénéficient les groupes andins et amazoniens.

Álvarez (2002) et Arauz (1999) réfutent la thèse de l'acculturation et parlent plutôt d'une stratégie développée par ces groupes dans le contexte de la colonisation espagnole pour la survie collective. Ladoption des us et coutumes de la société dominante et l'usage de l'espagnol leur permettraient de se camoufler pour pouvoir exister et survivre comme entité collective (Álvarez 2002 : 112; Arauz 1999). Il s'agit d'une forme d'infrapolitique des subalternes par laquelle certains groupes habillent leur résistance de
Tableau 5

Pourcentage des personnes s'auto-identifiant comme indigènes dans la région littorale, 2010

\begin{tabular}{|l|c|}
\hline Provinces & INDIGĖNES \\
\hline Manabi & 0,2 \\
\hline Santa Elena & 1,4 \\
\hline Guayas & 1,3 \\
\hline Esmeraldas & 2,8 \\
\hline El Oro & 0,7 \\
\hline Los Rios & 0,6 \\
\hline \multirow{2}{*}{ (Source : élaboration de l'auteure à partir des données de I'INEC 2012) } \\
\hline
\end{tabular}

rituels de subordination afin de déguiser leurs objectifs et d'atténuer les conséquences d'un éventuel échec (Scott 2009: 111). Les discours et les pratiques de résistance jouent ainsi le rôle de soupape de sécurité.

Roberts rejoint Álvarez et Arauz en mentionnant que, dans le contexte du boom du cacao, les groupes du littoral méridional ne montraient aucun intérêt à aller travailler dans les plantations. Ils préféraient rester dans une situation d'isolement volontaire en subvenant à leurs besoins par la chasse, la pêche et l'agriculture de subsistance. Selon elle, c'est une stratégie intentionnelle pour maintenir la séparation physique et culturelle avec les Blancs (Roberts 2010 : 54).

\section{Ethinicisation dans le CADRE Des luttes POUR L'ACCÈS À LA TERRE}

Pour contextualiser les conflits actuels relatifs à la vente des parcelles dans la région méridionale du littoral, il est indispensable de se référer aux caractéristiques de la propriété de la terre, aux impacts de la Loi des communes promulguée en 1937 et à la portée limitée des réformes agraires (1964 et 1973).

La production cacaoyère du littoral méridional était caractérisée par des plantations étendues sur de vastes territoires qui concentraient les rivières navigables. Cette structure était héritée de l'époque coloniale, durant laquelle la propriété des terres était attribuée à ceux qui les occupaient 
pacifiquement et continuellement pendant trente années consécutives. Les hacendados pouvaient élargir périodiquement leurs territoires en intégrant les parcelles qui se trouvaient à proximité. Ainsi, les limites entre la propriété privée et la propriété publique du littoral méridional étaient souvent confuses et difficiles à démontrer légalement (Roberts 2010 : 21-22). Progressivement, les frontières agricoles se sont étendues au détriment des groupes qui n'avaient pas de statut légal ou n'avaient qu'un statut précaire (Deler 2007 : 277).

La Loi des communes de 1937, une loi d'aménagement du territoire, visait la réorganisation, la gestion, le contrôle et l'unité du territoire national. Elle ne reconnaissait ni l'existence des communautés indiennes, ni leurs territorialités, ni la propriété collective de la terre (Massal 2005 : 183). Même si certaines formes particulières de production économique et de propriété collective étaient reconnues et protégées, il s'agissait plutôt de la volonté de les adapter au système juridique en place, en conformité avec les principes du système économique international (Alvarez 2002 : 10). Son effet principal dans la région d'étude a été la fragmentation des territoires historiquement très étendus. Cette fragmentation a été possible par leur parcellisation et par l'extension des droits de propriété privée favorisant les stratégies familiales au détriment des stratégies communautaires. Il est devenu plus facile pour les acteurs externes de diviser l'unité des familles formant la commune, pour des raisons économiques (ibid. : 27). Un des effets de cette loi a été la sédentarisation de certains groupes comme les Tsachilas.

Parallèlement, à partir des années 40 , les activités agricoles axées sur l'exportation se sont diversifiées, notamment par la culture de la banane-fruit qui a exacerbé la tendance des migrations des régions andine et amazonienne vers la région littorale. En conséquence, il y a eu une montée des demandes des groupes économiques métis et blancs pour l'acquisition des terres. Ces derniers remettaient en question la légalité de certaines terres communales, principalement celles de la province de Manabi et ils n'hésitaient pas à recourir aux tribunaux pour contester le caractère communal de certaines parcelles. À partir des années 1950, la convoitise des entrepreneurs pour les terres de la région et la remise en cause de l'identité indienne de ces groupes et du caractère communal des terres ont augmenté la conflictualité dans la région. Dans ce contexte, la Fédération des communes de Guayas a été fondée en 1965 pour la défense de la propriété communale.

Quant aux réformes agraires de 1964 et de 1973, elles ont été tardives en Équateur comparativement à d'autres pays latino-américains. Les revendications se sont concentrées dans la région andine en raison des impacts différenciés de la pression démographique sur l'accès aux terres cultivables et les ressources hydriques, de l'atomisation des structures agraires et de la présence des organisations syndicalistes de gauche (Sánchez Parga 2007). C'est la région amazonienne qui a subi les effets collatéraux des réformes puisque plusieurs projets de colonisation agricole y ont été entrepris dans les années 70 par l'Institut équatorien de réforme agraire et de colonisation en vue d'éponger le trop plein des agriculteurs andins, mais aussi littoraux (Collin Delavaud 1981: 355) liés aux dynamiques émergentes mentionnées précédemment.

Par la suite, dans les années 90, certains projets de construction (barrages, canaux d'irrigation) mis en œuvre par les autorités étatiques pour contrer la problématique de sécheresse dans la péninsule de Santa Elena, ont eu des impacts sur les communautés vivant dans les environnements immédiats. Ces projets ont favorisé l'intérêt des entrepreneurs pour les terres communales situées dans le secteur (Bazurco Osorio 2006: 135). Ce processus a engendré l'achat d'une importante quantité de parcelles communales avec possibilité d'irrigation par les acteurs du secteur privé. Cependant, ceux-ci ne visaient pas tous la production agricole, mais aussi la spéculation des terres (Espinel et Herrera 2008 : 33).

Durant la présidence de Rafael Correa (2006-2017) avec le passage d'un modèle économique néolibéral au modèle « extractiviste-exportateur », l'État est devenu un acteur central dans la gestion économique et dans l'exploitation des ressources naturelles (Svampa 2011). La problématique de la vente des parcelles communales s'est exacerbée, notamment, par l'impossibilité d'avoir accès à des crédits ou à des subventions étatiques sans titre de propriété privée (Álvarez 2016 : 334). Pour le cas de Manabi, selon les habitants de la commune (comuneros) de certaines terres communales situées entre les villages de Macaboa et de Río Piedra, la vente était illégale sans le consentement de tous les habitants de la commune, alors que l'État défendait sa légalité en argumentant que ceux-ci ne se sont pas ouvertement identifiés comme Indiens (Bauer 2009 : 179) et que les terres en question ne constituaient pas des territoires ancestraux.

Dans le cas de la péninsule de Santa Elena, l'administration Correa a accéléré les projets de construction de barrages et de canaux d'irrigation toujours dans le but de rendre les terres arides propices à l'agriculture. Face aux menaces d'achat des parcelles par le secteur privé, les habitants des communes se sont basés sur l'article 57 de la Constitution de $2008^{7}$ pour prouver la thèse d'illégalité des ventes. De plus, après la provincialisation de Santa Elena, les communes de la Péninsule ont fondé en 2010 la Fédération des communes de Santa Elena. Cette organisation assure un soutien juridique aux communes en situation de conflit et un accompagnement pour favoriser les capacités organisationnelles (Tuaza et Ozaetta 2014 : 27).

Certains journalistes traitent de ces conflits en déplorant la montée de la conflictualité dans la région et l'invasion des terres communales par les acteurs exogènes, souvent au moyen de pratiques qu'ils qualifient de frauduleuses (Calero Larrea 2016). Ils attribuent l'émergence de ces conflits aux politiques permissives de l'État, lesquelles laissent place à la spéculation des terres dans la région et au nonrespect des normes environnementales en agriculture et en aquaculture. D'une part, cette prise de position étatique pour ne pas reconnaître les droits ancestraux de certains comuneros, facilite l'exploitation des ressources. D'autre part, les organisations communales ont commencé à établir des liens avec les organisations indigènes régionales et 
nationales (Confédération des nationalités indigènes de l'Équateur) et avec les organisations non gouvernementales internationales pour la protection des droits territoriaux et la préservation des ressources naturelles (p. ex. les mangroves)

Bauer (2009) qualifie ce processus de réautochtonisation. En adoptant cette stratégie, les groupes du littoral méridional se sont joints tardivement au mouvement social indigène qui s'est structuré dans le pays à partir de la fin des années 1970. Les leaders des communes qui s'opposaient à la vente des terres collectives et qui étaient conscients du processus de la structuration d'un mouvement politique à l'échelle nationale ont commencé à formuler leur propre lutte comme étant une lutte ethnique (ibid. : 179).

\section{LE CAS DU CHANGEMENT DU NOM DE LA ROUTE DU SOLEIL POUR LA « ROUTE DU SPONDYLUS "}

Le secteur touristique se trouve à la croisée de deux phénomènes importants. D'une part, le potentiel touristique de la région a renforcé la tendance à la fragmentation des structures foncières et à l'intervention des acteurs externes (p. ex. ventes des parcelles aux investisseurs touristiques, pratiques de location des parcelles à des fins touristiques). D'autre part, il n'échappe pas à la tendance interventionniste dans le domaine économique caractérisant l'administration Correa.

Les données ethnologiques de Ruiz Ballesteros (2009) sur le tourisme communautaire dans la commune d'Agua Blanca démontrent l'imbrication des activités touristiques et des revendications identitaires. Comme Bauer, Ballesteros parle aussi de la récupération du passé préhispanique dans un contexte où les communes se voient dans l'obligation de certifier leur identité. Il s'agit notamment d'extérioriser certaines caractéristiques jusque-là non extériorisées ou de miser sur des configurations culturelles conformes aux représentations hégémoniques de l'autochtonie. À titre d'exemple, il est possible d'évoquer la participation de certains habitants des communes au festival de la balsa manteña comme rituel de réaffirmation identitaire et culturelle (Ruiz Ballesteros 2009 : 82).

Parallèlement, dans le contexte de compétition entre les investisseurs, l'État et les habitants des communes pour l'accès et l'administration des territoires de cette région à fort potentiel touristique, les autorités équatoriennes ont élaboré le Plan de marketing touristique de l'Équateur 2008-2020. Dans l'ensemble, ce plan vise à promouvoir le pays comme une destination touristique mondiale dans le respect des principes du tourisme durable. La stratégie adoptée pour la région d'étude est la restructuration de certains produits touristiques (Parc national Machalilla, Puerto Lopez, Isla de la Plata, etc.) sous forme de paquet multi-activités (Plandetur 2020, in MTE 2008 : 16). De plus, le Plan classifie les produits touristiques selon les catégories : étoile (p. ex. îles Galapagos), A (p. ex. Quito, Otavalo) et B, ce dernier correspondant aux produits à bas potentiel d'attraction touristique qui doivent être jumelés à d'autres de catégorie A ou étoile. Les sites abritant les restes archéologiques des civilisations préhispaniques du littoral comme l'île de la Plata (berceau de la culture valdivia) ou le Parc national de Machalilla sont conçus comme des produits complémentaires aux beautés naturelles paysagères du littoral. Dans cette même logique, les autorités équatoriennes ont changé le nom du circuit touristique de la route du Soleil, longeant les côtes des provinces de Manabi et de Santa Elena et concentrant les plus belles plages du pays, par « route du Spondylus ».

Il s'agit d'un changement majeur dans les stratégies de marketing touristiques puisque l'appellation « route du Soleil » faisait davantage référence aux beautés naturelles et paysagères de la région comme attraits touristiques alors que l'appellation « route du Spondylus » fait référence avant tout au patrimoine culturel et archéologique de la région. La route du Spondylus suit l'ancienne trajectoire du commerce de spondylus utilisée par les ancêtres des Mantas-Huancavilcas.

Cette situation met en évidence les ambiguités dans les attitudes étatiques au sujet de l'autochtonie des groupes du littoral méridional. En combinant ces deux études de cas, il s'avère que le passé autochtone de la région littorale est reconnu et fait même partie des stratégies de marketing touristique. Cependant, quand il s'agit des revendications appartenant au présent, les terres communales de ces mêmes groupes ne constituent pas des territoires ancestraux.

\section{Conclusion}

Linvisibilisation des Mantas-Huancavilcas dans les politiques coloniales et républicaines se situe dans un processus historique où le littoral se situait à la périphérie du centre politique et de la cosmovision inca. À l'époque coloniale, le statut périphérique du littoral a perduré jusqu'à ce que la production cacaoyère devienne la colonne vertébrale de l'économie de l'Audience royale vers la fin du XviII' siècle. Cependant, la situation périphérique du littoral et la 
place marginale de ses Indiens dans la main-d'œuvre totale disponible n'ont pas engendré leur absence dans les données sociodémographiques de l'époque coloniale. Au sein de la jeune république, l'invisibilisation des MantasHuancavilcas est d'abord observée par leur non-considération dans l'implantation des missions religieuses mandatées pour hispaniser et évangéliser les groupes situés en périphérie. Ladoption des politiques indigénistes à partir des années 1930 constitue un point tournant dans la structuration des représentations différenciées relatives aux groupes andins, amazoniens et littoraux. Dans cette période, les élites politiques étaient hantées par la façon dont les Indiens andins, nombreux, pauvres et économiquement improductifs, seront intégrés à la nation et à l'économie équatorienne. Les Indiens du littoral, sans marqueurs identitaires visuels tels que le code vestimentaire et l'usage d'une langue indienne, ne présentaient pas de défi particulier.

Au fur et à mesure que les critères socioculturels se sont introduits dans les questionnaires de recensement, commençant par la langue d'usage, l'indianité des MantasHuancavilcas s'est effacée de plus en plus. Comme le souligne la documentation spécialisée sur l'autochtonie du littoral, les habitants des communes ne s'identifiaient pas aux catégories proposées dans les questionnaires du recensement. Historiquement, leurs membres ne se sont pas explicitement identifiés comme indiens ou indigènes. Au contraire, selon les données ethnographiques, ces groupes ont délibérément opté pour l'invisibilisation afin d'assurer leur survie et leur reproduction biologique et culturelle. À tout cela s'ajoute l'attention portée par le monde académique et la presse nationale et internationale sur les mobilisations des groupes andins et amazoniens durant les années 1990 et 2000.

À partir de la fin des années 60, une série de phénomènes a mis en évidence les limites de la stratégie volontaire d'invisibilisation comme pratique de résistance et la nécessité de structurer un discours identitaire différent afin de protéger les terres communales. Il s'agit de l'émergence des conflits relatifs au statut légal des terres communales, des enjeux de fragmentation et de vente des terres et du développement des activités touristiques dans la région. Dans ce nouveau contexte, l'exhibition de l'autochtonie et l'actualisation de certaines pratiques identitaires deviennent en quelque sorte des obligations pour certifier ou authentifier l'ancestralité et pour assurer la reconnaissance de certains droits.

\section{Notes}

1. Le terme cholo a plusieurs significations en Amérique latine en fonction des pays ou des contextes. Il peut aussi être péjoratif ou affectueux. Dans le cadre de cet article, il réfère à l'identité autochtone acculturée du littoral équatorien.

2. Attribution par la Couronne espagnole aux premiers colonisateurs espagnols et à leurs descendants des parcelles de terres et un certain nombre d'autochtones tributaires.

3. Comme les Awas et les Eperas, originaires de la Colombie, l'ont quittée en raison de la guerre entre les libéraux et les conservateurs (1899 et 1902) et de la violence perpétrée par les guérillas et les groupes paramilitaires colombiens, ils ne figurent pas dans les croquis de cette période.

4. Expression utilisée durant la période d'esclavage dans les Amériques (Louisiane, îles de la Caraïbe, Haiti) pour désigner les personnes de descendance mixte africaine-européenne qui n'étaient pas des esclaves.

5. En Équateur, les termes «nationalité » et «peuple » réfèrent à deux types de démarcation identitaires en fonction d'expériences historiques propres. Un peuple indigène se définit comme tel en relation à une société qui n'est pas originelle et ne peut le devenir, du fait de la conscience que développent ses membres sur cette situation. La nationalité, quant à elle, englobe les peuples autochtones ayant une même origine, une histoire commune, des langues propres et régis par leurs propres lois, coutumes, croyances, formes d'organisation sociale, économique et politique dans leurs territoires. Il existe quatorze nationalités et quinze peuples dans le pays.

6. La loi des communes de 1937 stipule que tout centre de population inférieur à la catégorie de paroisse et qui ne soit pas une des catégories existantes (caserío, anejo, barrio, partido, parcialidad, comunidad) sera désormais nommé « commune». La commune n'est pas exclusivement une forme d'organisation politique et sociale indienne. Elle est plutôt une unité de la structure politicoadministrative de l'État. Pour pouvoir être éligible à sa formation, il fallait être au moins cinquante habitants et avoir le contrôle des terres revendiquées depuis au moins trente ans. Il n'existe donc aucun critère relatif à l'ethnie ou à la langue parlée pour la formation d'une commune.

7. L'article en question énumère une série d'objectifs et de dispositions pour protéger les droits collectifs des communes, des communautés et des peuples ou nationalités indigènes.

\section{Ouvrages cités}

ÁLVAREZ, Silvia, 1997 : « Cronicas desde el mar: una aproximación a la condición indígena en la costa ecuatoriana», in José Juncoso et al. (dir.), Etnografias minimas del Ecuador. TsachilaChachis-Cholo-Cofán-Awa-Coaiquer: 89-116. Abya Yala, Quito.

—, 2002: «De reducciones a comunas: transformaciones legales de las tierras comunales en la península de Santa Elena, Ecuador». Quaderns de l'Institut Català d'Antropologia 17-18 : 7-43.

_, 2016: «La importancia de tener nombre: Identidad y derechos territoriales para las comunas de Santa Elena». Ecuador, Antropología Experimental 16:325-352.

ANGOSTO FERNANDEZ, Luis, et Sabine KRADOLFER, 2012: Everlasting Countdowns: Race, Ethnicity and National Censuses in Latin American State. Cambridge Scholars Publishing. Cambridge.

ÁQUILES PÉREZ, Ramón, 1943: Así han vivido nuestros indios. Ministerio de Previsión Social y Trabajo, Quito.

AUSTIN ALCHON, Suzanne, 1991 : Native society and disease in colonial Ecuador. University of Cambridge University Press, Cambridge.

ARAUZ, Maritza, 1999 : Pueblos indios de la costa ecuatoriana. Jipijapa y Montecristi en la segunda mitad del siglo XVIII. Abya Yala, Quito.

BARRE, Marie-Chantal, 1983: Ideologias indigenistas y movimientos indios. Siglo XXI, Mexico.

BAUER, Eric, 2009: « Re-articulating identity: the shifting landscape of indigenous politics and power on the Ecuadorian coast ». Bulletin of Latin American Research 29(2) : 170-186.

BAZURCO OSORIO, Martin, 2006 : Yo soy más indio que tú: resignificando la etnicidad: exploración teórica e introducción al proceso de reconstrucción étnica en las Comunas de la Península de Santa Elena. Abya Yala, Quito.

CALERO LARREA, Carolina, 2016: "Y la herencia cultural, ia quién le importa?» La Barra espaciadora. <http://www.labarraespaciadora.com/pulso/y-la-herencia-cultural-a-quien-leimporta/> (consulté le 12 août 2016).

CHÁVEZ, Leopoldo, 1943 : «Educación de espíritu indígena », in Jorge Leon Trujillo et Simón Espinosa (dir.) Indianistas, Indianofilos, indigenistas : 551-582. ILDIS, Abya Yala, Quito.

CHÁVEZ GONZÁLEZ, Rodrigo, 1935 : « Estudios de idiosincrasia regional », in Jorge leon Trujillo et Simón Espinosa (dir.), Indianistas, Indianofilos, indigenistas: 291- 373. ILDIS, Abya Yala, Quito. 
CHIRIBOGA, Leonardo, 1939: «Problema del Indio examinado desde el punto de vista de la organización militar », in Jorge Leon Trujillo, Jorge et Simón Espinosa (dir.), Indianistas, Indianofilos, indigenistas : 583-638. ILDIS, Abya Yala, Quito.

COLLIN-DELAVAUD, Claude, 1981 : « Les gisements de pétrole du Piémont andin : fronts pionniers ou enclaves? Les cas du Pérou et de l'Équateur », in Pierre Monbeig (dir.), Le phénomène de frontière dans les pays tropicaux : table ronde organisée en l'honneur de Pierre Monbeig : 347-356. IHEAL, Paris.

COLLOREDO MANSFIELD, Rudi, 1998: «Dirty Indians, Radical Indígenas, and the Political Economy of Social Difference in Modern Ecuador ». Bulletin of Latin American Research 17(2): 185-205.

DELER, Jean-Paul, 2007 : Ecuador. Del espacio al Estado nacional. Universidad andina Simón Bolivar. IFEA, Corporación Editora nacional, Quito.

DELGADO CABRERA, Miguel 2011 : Aproximación a los conflictos en tierras comunales de la Península de Santa Elena. <http://www. ballenitasi.org/2011/11/aproximacion-los-conflictos-entierras.html> (consulté le 20 juin 2017).

ESPINEL, Ramon, et Paul HERRERA, 2008: «Acumulación perversa: Comuneros, agua y tierra en la Península Santa Elena », in Frank Brassel et al. (dir.), ¿Reforma agraria en el Ecuador? Viejos temas, nuevos argumentos : 49-64. SIPAE, Quito.

ESVERTIT COBES, Natalia, 2008 : La incipiente provincia. Amazonia y estado ecuatoriano en el siglo XIX. Universidad andina Bolivar/ Corporación nacional, Quito.

FAURÍA, Carmen, 1995 : «Particularidades de las encomiendas en la costa septentrional andina », in Aurelio Álvarez et al. (dir.), Primer encuentro de investigadores de la Costa ecuatoriana en Europa : 387-398. Abya Yala, Quito.

FAVRE, Henri, 1996 : L’indigénisme. Que sais-je? PUF, Paris.

GUAMAN POMA DE AYALA, Felipe, 1615 [?] : La nueva coronica y el buen gobierno. <http://www.kb.dk/permalink/2006/poma/info/ en/frontpage.htm> (consulté le 13 janvier 2016).

HAMERLY, Michael, 1973 : Historia social y económica de la Antigua provincia de Guayaquil 1763-1842. Publicaciones del Archivo histórico del Guayas, Guayaquil.

INEC (Instituto nacional de estadísticos y censos), 2006: « La población indígena del Ecuador ». <http://inec.gob.ec/inec/ index.php?option=com_remository\&Itemid $=420 \&$ func $=$ start down\&id=1031\&lang=es> (consulté le 24 juin 2016).

—, 2012 : « Résultats du recensement de $2010 » .<$ http://www.inec. gob.ec/cpv/descargables/fasciculo_nacional_final.pdf> (consulté le 3 septembre 2012).

—, 2015: «Evolución de las variables investigadas en los censos de población y vivienda 1950-2010». <http://www.ecuadorencifras. gob.ec/documentos/web-inec/Publicaciones/Evolucion_variables_1950_2010_24_04_2014.pdf> (consulté le 9 mai 2018).

JARAMILLO, ALVARADO, Pío, 1941 : «Situación política, económica y jurídica del indio en el Ecuador », in Jorge Trujillo et Simón Espinosa (dir.), Indianistas, Indianofilos, indigenistas: 453-494. ILDIS, Abya Yala, Quito.

KNAPP, Gregory, 1987: Geografia quichua de la Sierra del Ecuador. Ediciones Abya Yala, Quito.

LAVIANA CUETOS, Maria Luisa, 1987 : Guayaquil en el siglo XVIII. Recursos naturales y desarrollo económico. Escuela de Estudios hispano-americanos de Sevilla-Consejo Superior de investigaciones científicas, Sevilla.

LEÓN BORJA, Dora, 1988 : « Los Indios balseros como factor en el desarrollo del puerto de Guayaquil », in Jenny Estrada (dir.), La Balsa en la historia de la navegación ecuatoriana. Compilación de crónicas, estudios, gráficas y testimonios: 281-311. Instituto de Historia marítima, Guayaquil.

MACHADO, Decio, 2011a : « Historia de despojo en la provincia de Santa Elena. Historia de despojo y rapiña sobre las tierras comunales en la provincia de Santa Elena (Ecuador)». La Línea de Fuego, 12 septembre. <https://lalineadefuego.info/2011/09/12/ historia-de-despojo-en-la-provincia-de-santa-elena-por-deciomachado/> (consulté le 12 août 2016).
—, 2011b : «Problemática actual de las tierras comunales de Santa Elena». <http://www.ballenitasi.org/2011/09/problematicaactual-de-las-tierras.html> (consulté le 20 juin 2017).

MARTÍNEZ, Nicolas, 1916 : « La condición actual de la raza indígena en la provincia del Tungurahua », in Jorge Leon Trujillo et Simón Espinosa (dir.), Indianistas, Indianofilos, indigenistas: 207-244. ILDIS, Abya Yala, Quito.

MASSAL, Julie 2005: Les mouvements indiens en Équateur. Mobilisations protestataires et démocratie. Karthala, Paris.

MTE, 2008: Plan estratégico de desarrollo, de turismo sostenible para Ecuador (PLANDETUR) 2009-2020. Quito.

PAREJA, Miguel, et Willington PAREDES, 2006 : Identidad guayaquileña. Referentes socioculturales de aproximación. Archivo histórico de Guayas, Guayaquil.

PAZ Y MIÑO, Luis, 1942 : La población del Ecuador. Talleres gráficos de educación. Quito.

PORTAIS, Michel, 1990 : «La distribución demográfica de la población y su evolución », in D. Delaunay et al. (dir.), Transición demográfica en el Ecuador : 57-75. Instituto panamericana de geografía e historia, Quito. <http://horizon.documentation.ird.fr/exl-doc/ pleins_textes/doc34-07/34503.pdf> (consulté le 10 mai 2018).

ROBERTS, Lois, 2010: Ecuador en la época cacaotera. Respuestas locales al auge y colapso en el ciclo monoexportador. Corporación para el desarrollo de la educación universitaria, Codeu, Quito.

RUIZ BALLESTEROS, Esteban, 2009: Agua Blanca. Comunidad y turismo en el Pacifico ecuatorial. Abya-Yala, Quito.

SAINTOUL, Catherine, 1988 : Racismo, Etnocentrismo y Literatura: la Novela Indigenista Andina. Del Sol, Buenos Aires.

SALAZAR SOLER, Carmen, 2013 : « Reflexiones sobre estas categorías sociales en el Perú andino ». Nuevo Mundo Mundos Nuevos. <http:// nuevomundo.revues.org/66106> (consulté le 24 juin 2017).

SÁNCHEZ PARGA, José, 1996: Población y pobreza indígenas. Centro andino de acción popular, Quito.

—, 2007 : El movimiento indígena ecuatoriano. La larga ruta de la comunidad al partido. Centro andino de acción popular, Quito.

SAUNDERS, John, 1961 : The people of Ecuador. A demographic analysis. University of Florida Press, Gainesville.

SCOTT, James, 2009: La domination et les arts de la résistance. Fragments du discours subalterne. Éditions Amsterdam, Paris.

SINARDET, Emmanuelle, 1999a : «Léducation équatorienne et la révolution julienne (1925-1931). Rupture ou continuité?» Bulletin de l'Institut français d'études andines 28(1) : 123-157.

—, 1999b : «La preocupación higienista e la educación ecuatoriana en los años treinta y cuarenta ». Bulletin de l'Institut français des études andines 28(3): 411-432.

STOCKS, Anthony W., 1978: The invisible Indians: A history and analysis of the relations of the Cocamilla Indians of Loreto, Perú, to the State. Ph.D. Thesis, University of Florida, Florida. <http:// ufdc.ufl.edu/AA00004914/00001> (consulté le 10 mai 2018).

SVAMPA, Maristella, 2011 : "Néo-développementisme" extractiviste, gouvernements et mouvements sociaux en Amérique latine». Problèmes d'Amérique latine 81 : 103-127

TAYLOR, Anne Christine, 1991 : « Équateur : les Indiens de l'Amazonie et la question ethnique ». Problèmes d'Amérique latine 3: 109-122

TYRER, Robson Brines, 1988 : Historia demográfica y económica de la Audiencia de Quito. Población indígena e industria textil 16001800. Banco central del Ecuador, Quito.

TUAZA, Luis Alberto, et Carlos OZAETTA, 2014 : « Federación de las comunas de la provincia de Santa Elena: capacidad de agencia y conflictos ». Ciencias pedagógicas e innovación $2: 1$.

VENTURA I OLLER, Monserrat, 2009 : Identité, cosmologie et chamanisme des Tsachila de l'Équateur. Harmattan, Paris.

WACHTEL, Nathan, 1971 : La vision des vaincus. Les Indiens du Pérou devant la Conquête espagnole 1530-1570. Gallimard, Paris.

WOLF, Eric, 1962 : Peuples et civilisations de l'Amérique centrale. Des origines à nos jours. Payot, Paris. 